\title{
Miniature Engineered Tapered Fiber Tip Devices by Focused Ion Beam Micromachining
}

\author{
Fei $\mathrm{Xu}$, Jun-long Kou, Yan-qing Lu and Wei Hu \\ College of Engineering and Applied Sciences and \\ National Laboratory of Solid State Mi-crostructures, \\ Nanjing University, Nanjing, \\ P. R. China
}

\section{Introduction}

Optical fibers have been the basis of the modern information technology since Kao and Hockham proposed glass waveguides as a practical medium for communication in 1965. A lot of different optical fiber active/passive devices including couplers, interferometers, gratings, resonators and amplifiers have been widely employed for applications on telecommunications and sensing networks (Agrawal, 2002). For a number of applications, it is important to reduce the device's size. Small size is often attractive for particular sensing applications because of some benefits such as fast response to detecting small objection with little perturbation on the object being measured. There are two steps to obtain fiber devices as small as possible. First, it is to taper or etch the fiber and reduce its diameter. A subwavelength-scale microfiber is the basic element of miniature fiber devices and subsystems (Tong et al., 2003; Brambilla et al., 2004, 2005, 2010). The second is to engineer the microfiber to realize miniature version of conventional fiber devices. There are various fabrication methods to engineer the microfiber, such as $\mathrm{CO}_{2}$ laser, femtosecond (fs) laser, $\mathrm{HF}$ acid etching, arc splicing and focused ion beam (FIB). Most of these techniques have the difficulties in carving the microfiber freely because of the resolution. The latest progress in FIB technique has opened a new widow for ultra-small size fiber devices. So far, FIB is the most flexible and powerful tool for patterning, cross-sectioning or functionalizing a subwavelength circular microfiber due to its small and controllable spot size and high beam current density.

FIB systems have been produced commercially for approximately thirty years, primarily for large semiconductor manufacturers. FIB systems operate in a similar fashion to a scanning electron microscope (SEM) except, rather than a beam of electrons and as the name implies, FIB systems use a finely focused beam of ions that can be operated at low beam currents for imaging or high beam currents for site specific sputtering or milling (http://en.wikipedia.org/wiki/Focused_ion_beam). The fine and controllable ion spot size and high beam current density are perfect for micro- and nano-fabrications with high spatial resolution $(\sim 10 \mathrm{~nm})$. As a result, FIB has recently become a popular candidate for fabricating high-quality micro-devices or high-precision microstructures. Originally, FIB processing was used for mask repair (Liang et al., 2000), integrated circuit chip repair/modification (Liu et al., 2006), cross-sectional imaging of critical parts of 
semiconductor devices and sample preparation for transmission electron microscopy (Daniel et al., 1998; Hopman et al., 2008; Jeon et al., 2010). Besides these applications, FIB milling can also be used to assist carbon nanotube growth and manipulation (Hofmann et al., 2005; Deng et al., 2006), pattern magnetic data storage media (Terris et al., 2007) and structure hard-to-etch materials like $\mathrm{SiC}$ or $\mathrm{LiNbO}_{3}$. In the field of optoelectronics, there have been extensively studies toward utilizing the FIB as a machining tool to fabricate planar micro-optical components with low surface roughness for integrated optical circuits, for example, the end facet mirrors, ring resonators, gratings and photonic crystals (Hopman et al., 2008). Obviously, FIB processing can and in fact has been widely applied to fabricate microfiber based devices to reduce the size of fiber devices as much as possible.

In this chapter, we will review several kinds of ultra-small engineered tapered fiber tip (TFT) devices including interferometers and gratings by FIB micromachining and their characteristics and sensing applications.

\section{Fabrication and measurement}

Standard optical TFT is an optical microfiber with only one output or input end and a taper transition. The taper transition is connected to untapered fiber at the extremities which can easily be connected to other fiber optic components. The taper is etched or pulled from a standard single mode fiber when heated by a $\mathrm{CO}_{2}$ laser, electrical microheater or a small flame. Since the TFT is for analyte detecting rather than launching the light, it should be short enough in order to be rigid. However, too short and sharp shape results in high losses due to the poor 'adiabaticity' of the taper profile which couples light to lossy unbound modes (Love et al., 1991). During the last decade, much work has been carried out to study and optimize TFT profiles for telecom devices. Technology development allows manufacturing tapers with diameters well below $100 \mathrm{~nm}$ and it is possible to tailor the taper shape to an ideal profile (Brambilla \& Xu, 2007). The quickest and simplest way to manufacture short TFT relies on using a commercially available pipette puller. This method is often used to manufacture fibre tips for optical tweezers and scanning near-field optical microscopy (SNOM) tips. In this chapter, we make TFTs using a commercial pipette puller (model P-2000, Sutter Instrument). The P-2000 is a microprocessor-controlled $\mathrm{CO}_{2}$ laserbased micropipette puller. The bare fibre is held on two puller stages. The P-2000 can also be used to pull tubes and optical fibres to extremely small diameters. The pipette puller has five parameters which can be adjusted to achieve the wanted profile. The fabrication process is simple, convenient and extremely fast, which usually takes less than 0.3 second. The obtained TFT is then checked under a high-magnification optical microscope. Figure 1 shows a microscope image of a typical TFT with a sharp profile.

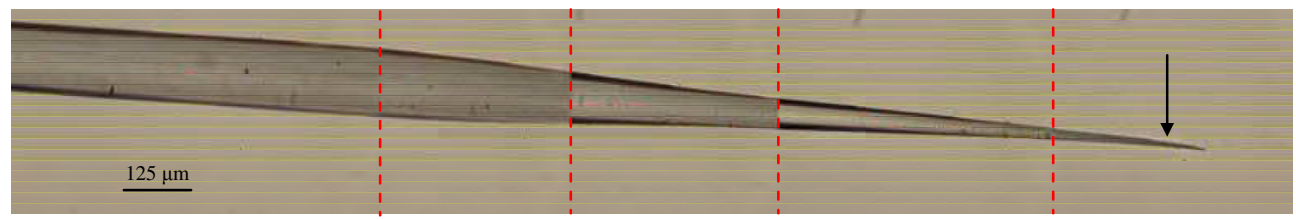

Fig. 1. Microscope image of a typical TFT, five photographs separated by four dashed vertical lines are used to show the whole profile of the TFT. The black arrow indicates the milling location (Kou et al., 2010b). Reprinted with permission. Copyright 2010 Optical Society of America 
The TFT is then coated with a thin metal layer such as aluminium (Al) or gold ( $\mathrm{Au}$, for exciting surface waves discussed in Section 4.2). The coating thickness is around $30 \sim 150$ $\mathrm{nm}$ and the metal is deposited on only one side of the taper. The metal Al is used as a conductive layer to prevent gallium ion accumulation in the FIB micromachining process. Then, the Al-coated TFT is placed stably in the FIB machining chamber (Strata FIB 201, FEI Company, Ga ions) using conductive copper tape. We generally use a $30.0 \mathrm{kV}$ gallium ion beam with current $60 \sim 300 \mathrm{pA}$. This enables us to make structures with high accuracy and sharp end-faces. We mill the structures from the taper end with small diameter to that with bigger diameter, because the milled part becomes non-conductive when the metal is removed by the beam. The total micro-machining process takes about $15 \sim 30$ minutes depending on the size of the machined structures. Finally, the TFT is immersed in hydrochloric acid for about $15 \sim 30$ minutes to totally remove the Al layer before cleaned with deionized water. In our experiment, the cavity or grating is made from a two-step process. Because there are some remains adhering onto the surfaces of the cavity after the first milling step, a second step under the same or smaller beam current is used to improve the surface smoothness.

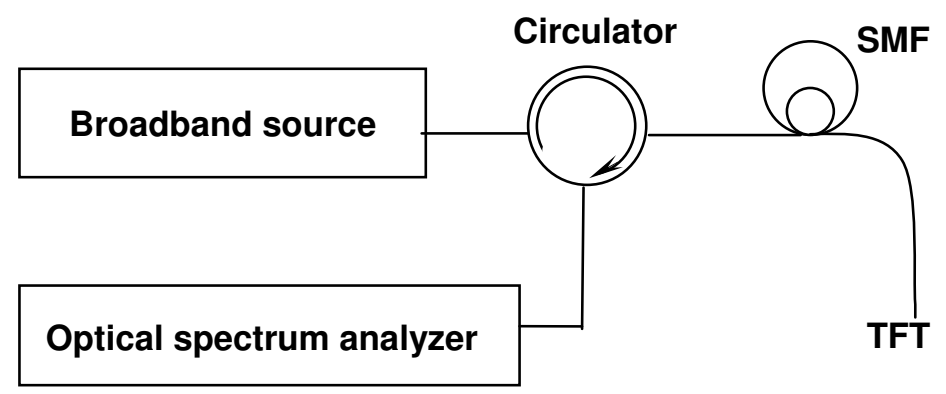

Fig. 2. Experimental setup of an FPMI.

In this chapter, we mainly consider the reflected signals. The reflective spectral response of these TFT based devices are measured with a broadband source $(1525 \sim 1610 \mathrm{~nm})$ and an Ando AQ6317B optical spectrum analyzer (OSA) through a circulator, as shown in Fig. 2. The TFTs before milling display an ignorable reflection of less than - $100 \mathrm{~dB}$ over the whole broadband spectrum.

\section{FIB machined micro-cavity TFT interferometers}

Optical fiber interferometers have been extensively used in various sensing applications due to its advantages of versatility, linear response and relatively simple structure. In the past decades, a lot of efforts have been made to develop intrinsic and extrinsic interferometers, especially the micro-cavity Fabry-Perot interferometers (MCFPIs). MCFPIs with tens-ofmicrometer-length cavity are attractive because of the small size, large free spectrum range (FSR) and high sensitivity. The cavity can be assembled by splicing two single mode fibers (SMFs) to a hollow-core fiber (Sirkis et al., 1993), inserting a silica SMF and a multi-mode fiber into a glass capillary (Bhatia et al., 1996), or splicing a SMF and an index-guiding photonic crystal fiber together (Villatoro et al., 2009). Although many progresses have been made, people are still pursuing new micro cavity fabrication techniques to improve the cavity length precision, structure accuracy and the process repeatability. Femtosecond laser 
technology thus was proposed recently showing great success in micromachining fiber devices. MCFPIs can be quickly fabricated by milling a small-open hole in a SMF for liquid and gas sensing (Rao et al., 2007). However, even the fs-laser machined MCFPIs still show low fringe visibility of several $\mathrm{dBs}$ in liquids due to the rugged surfaces inside the cavity; what's more, it is difficult to focus the laser spot to a sub-wavelength scale due to the diffraction limit. Thus the micromachining accuracy is limited and the size of the microcavity is large (tens of micrometers). The latest progress in FIB technique opens a new widow of opportunity for ultra-small size cavity (Kou et al., 2010a, 2010b). Microcavities with nanometer-scale accuracy in a subwavelength microfiber could be fabricated by FIB, which is relatively difficult for fs laser approach. There are several typical geometries which can be realized by FIB machined-TFT as shown in Fig. 3. Among them, an open-notch in one side is the most preferred and easiest to be fabricated.

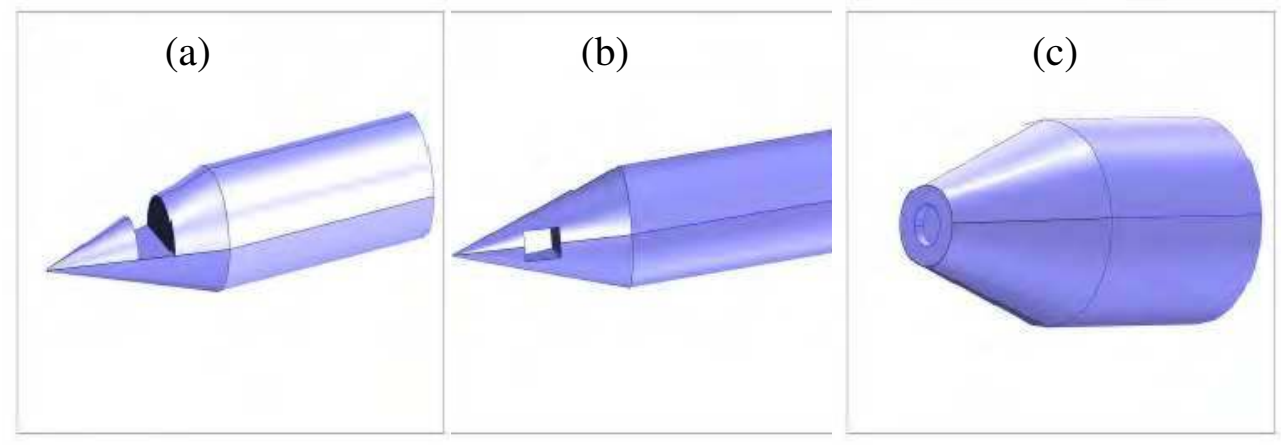

Fig. 3. Illustration of several typical geometries which can be realized by FIB machining, (a) a side open notch, (b) a hole in the middle and (c) a hole in the tip end and parallel to the fiber axis.

For geometry (a), due to the low reflectivity of the air-glass interfaces, multiple reflections have negligible contributions to the optical interference. However, a TFT consists of a SMF and a MMF in nature, without splicing. It may hold both the original single core mode and the multi-modes in the cladding at different positions. As shown in Fig. 4, we only consider two reflections $\mathrm{I}_{1}$ and $\mathrm{I}_{2}$ at the two end-faces, respectively. The fundamental $\mathrm{LP}_{01}$ mode can be coupled to high-order $\mathrm{LP}_{0 \mathrm{~m}}$ mode in the taper transition or be excited to high-order $\mathrm{LP}_{0 \mathrm{~m}}$ mode at the end-faces. $\mathrm{I}_{1}$ or $\mathrm{I}_{2}$ possibly includes $\mathrm{LP}_{01}$ or $\mathrm{LP}_{0 \mathrm{~m}}$ mode (Kou et al., 2010b). We also break the cavity and measure the reflection $I_{1}$ at end-face 1 ; flat reflective spectrum without obvious interference fringes is observed. Accordingly, a reasonable assumption is to consider only one dominated mode in $\mathrm{I}_{1}$ ( $\mathrm{LP}_{01}$ mode) and $\mathrm{I}_{2}\left(\mathrm{LP}_{01}\right.$ or $\mathrm{LP}_{0 \mathrm{~m}}$ mode excited when inputting $I_{2}$ into end-face 1 ) (Kou et al., 2010b). We call this kind of device as a hybrid FP modal interferometer (FPMI). The interference spectrum can be modelled using the following two-beam optical interference equation (Kou et al., 2010b):

$$
I=I_{1}+I_{2}+2 \sqrt{I_{1} I_{2}} \cos \left(\delta+\varphi_{0}\right)
$$

The phase difference between two modes in $\mathrm{I}_{1}$ and $\mathrm{I}_{2}$ is (Kou et al., 2010b) 


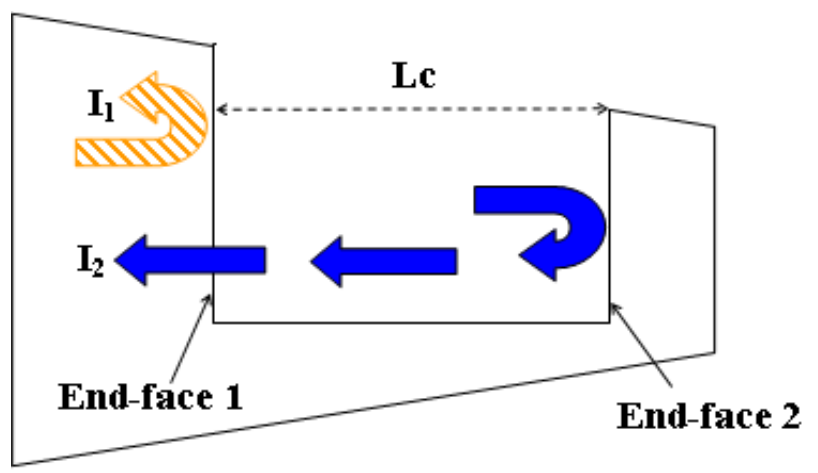

Fig. 4. Illustration of the FPMI. $I_{1}$ and $I_{2}$ are the reflections at end-face 1 and end-face 2 respectively; $\mathrm{L}_{\mathrm{c}}$ is the length of the cavity. When $\mathrm{I}_{2}$ enters end-face 1 , the fundamental mode is possible to be excited to a higher-order mode (Kou et al., 2010b). Reprinted with permission. Copyright 2010 Optical Society of America

$$
\left\{\begin{array}{l}
\delta=\delta_{1}+q \delta_{2}=(2 \pi / \lambda)\left(\Delta_{1}+q \Delta_{2}\right) \\
\Delta_{1}=2 n_{c} L_{c} \\
\Delta_{2}=\int\left(n_{2}(r)-n_{1}(r)\right) d z(r)
\end{array}\right.
$$

and FSR is (Kou et al., 2010b)

$$
\mathrm{FSR}=2 \Pi \lambda / \delta
$$

where $\mathrm{q}=0$ (for $\mathrm{LP}_{01}$ in $\mathrm{I}_{2}$ ) and 1 for $\left(\mathrm{LP}_{0 \mathrm{~m}}\right.$ in $\left.\mathrm{I}_{2}\right) ; \Delta_{1}\left(\delta_{1}\right)$ and $\Delta_{2}\left(\delta_{2}\right)$ are the optical path length difference (the phase difference) owing to the micro-cavity and the modal difference in the taper transition, respectively; $\mathrm{n}_{1}(\mathrm{r})$ and $\mathrm{n}_{2}(\mathrm{r})$ are the effective index of $\mathrm{LP}_{01}$ and $\mathrm{LP}_{0 \mathrm{~m}}$ modes, respectively, functions of local radius $\mathrm{r}(\mathrm{z})$ of the TFT at position $\mathrm{z}$, which can be calculated by three-layer model of finite cladding step-profile fiber with the TFT profile $r(z)$ which can be obtained from the microscope figure of the TFT (Kou et al., 2010b).

\subsection{FIB machined FPMI for temperature sensing}

The FPMI can be applied as a high-temperature sensor. Its extremely small size and especially unique structure offer great potentials for fast-response high temperature sensing particularly in small and harsh area with high temperature gradient, such as micro-flame and high temperature gas-phase/liquid-phase flow in microfluidics channel. Figure 5 shows an SEM picture of a FPMI with a micro-notch cavity from the side view and cross section after cleaving the TFT at the cavity. The end-face is very sharp and smooth. The cavity is 4.4 $\mu \mathrm{m}$ long and $5 \mu \mathrm{m}$ high, located at the position with the local radius $r=4.6 \mu \mathrm{m}$. 

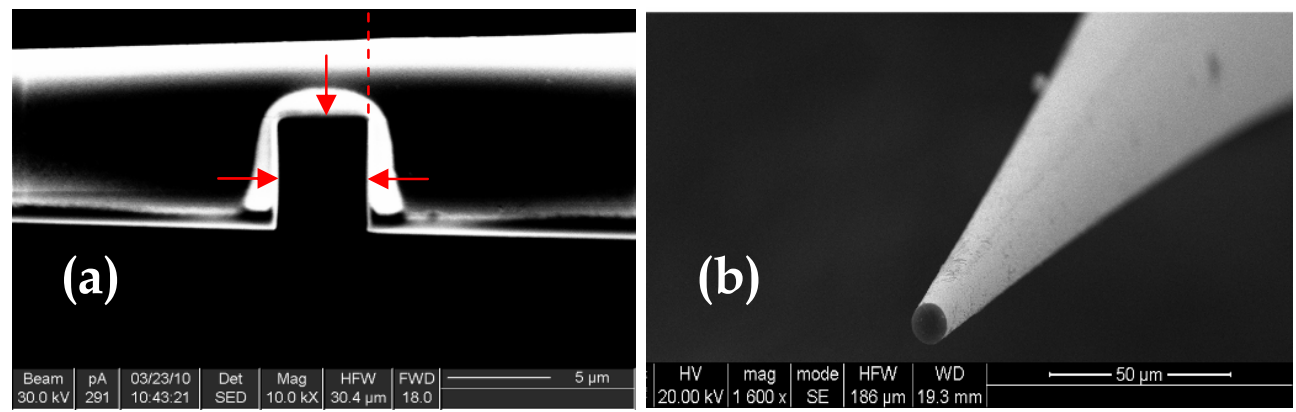

Fig. 5. SEM image (a) of the micro-notch cavity from the side view: three arrows show the edges of the cavity at the fiber tip, (b) of the cross section with the fiber tip cleaved at the position indicated in (a) by a dash line (Kou et al., 2010b). Reprinted with permission. Copyright 2010 Optical Society of America

The reflective spectral response of this FPMI device is measured with the setup as shown in Fig. 2. The TFT without a cavity displays an ignorable reflection of less than - $100 \mathrm{~dB}$ over the whole broadband spectrum. Hence, the detected signal is the light reflected only at the two end-faces of the micro-cavity, and the reflection at the tip end is negligible. The interference spectra of the FPMI device at room temperatures $\left(19^{\circ} \mathrm{C}\right)$ are shown in Fig. 6 . The spectra indicates a free spectral range (FSR) of $\sim 11 \mathrm{~nm}$ and a fringe visibility of $\sim 11 \mathrm{~dB}$ around $1550 \mathrm{~nm}$, which is larger than some other MCFPI sensors (Choi et al., 2008), and enough for sensing application. $\delta_{1}$ is $\sim 12 \Pi$ and $\delta_{2} \sim 295 \Pi$ for $L_{03}$ mode, and FSR $\sim 10 \mathrm{~nm}$, in good agreement with what we obtain in the experiment. In our calculation, $\lambda=1530 \mathrm{~nm}, \mathrm{~L}_{\mathrm{c}}$ $=4.4 \mu \mathrm{m}$ and $\mathrm{n}_{\mathrm{c}}=1$ (Kou et al., 2010b).

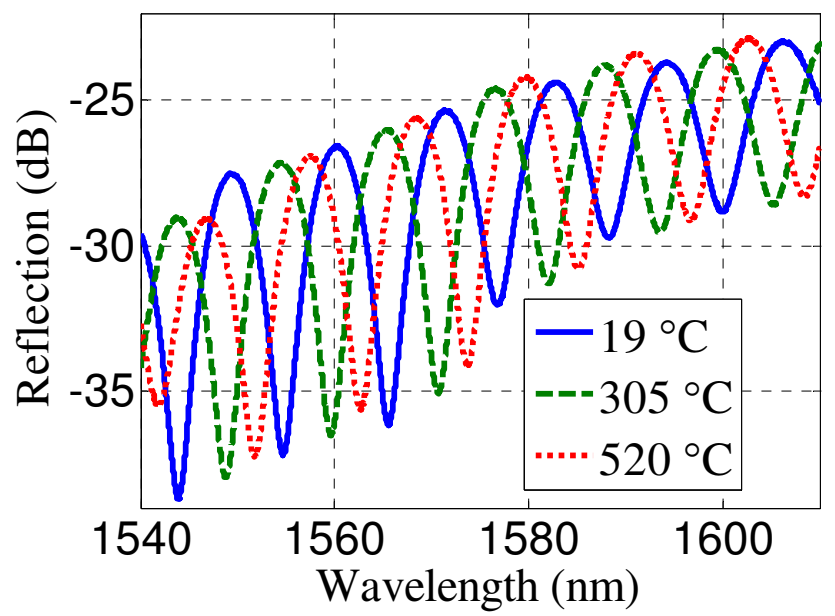

Fig. 6. Interference spectra of the FPMI device in air at different temperatures (Kou et al., 2010b) Reprinted with permission. Copyright 2010 Optical Society of America

We characterize the thermal response of the FTMI device by heating it up in a micro-furnace (FIBHEAT200, Micropyretics Heaters International Inc.) and temperature ranging from 
room temperature $\left(19^{\circ} \mathrm{C}\right)$ to $520^{\circ} \mathrm{C}$ is measured by a thermocouple (TES-1310, Type K, TES Electrical Electronic Corp.). The spectrum and temperature were recorded when both of them are stable for several minutes (Kou et al., 2010b).

The temperature sensitivity $\mathrm{S}_{\mathrm{T}}$ is defined as the interference wavelength shift divided by the corresponding temperature change. $\mathrm{S}_{\mathrm{T}}$ depends on temperature through the thermal expansion and/or thermo-optics effect (Choi et al., 2008; Kou et al., 2010b):

$$
\left\{\begin{array}{l}
S_{T}=\frac{d \lambda}{d T}=\frac{2 \pi}{\delta}\left(\frac{d \Delta_{1}}{d T}+\frac{d \Delta_{2}}{d T}\right)=\frac{2 \pi}{\delta}\left(2 \alpha_{T} L_{c}+\frac{d \Delta_{2}}{d T}\right) \\
\frac{d \Delta_{2}}{d T} \simeq \int\left[\frac{\partial\left(n_{1}-n_{2}\right)}{\partial n} \sigma_{T}+\frac{\partial\left(n_{1}-n_{2}\right)}{\partial r} \alpha_{T}\right] d z
\end{array}\right.
$$

where $\sigma_{\mathrm{T}}\left(1.1 \times 10^{-5} /{ }^{\circ} \mathrm{C}\right)$ is the thermo-optics coefficient and $\mathrm{a}_{\mathrm{T}}\left(5.5 \times 10^{-7} /{ }^{\circ} \mathrm{C}\right)$ is the thermal expansion coefficient. There are two contributions from temperature change: the temperature-induced length variation in the cavity, and the temperature-induced index variation and taper volume variation in taper transition. The first one is less than $1 \mathrm{pm} /{ }^{\circ} \mathrm{C}$ and ignorable, it agrees with the fact that those previous micro-cavity FP interferences in SMF by femtosecond laser machining are temperature-insensitive; the second one is about $10 \sim 20 \mathrm{pm} /{ }^{\circ} \mathrm{C}$ and dominates in temperature sensing (Kou et al., 2010b).

Figure 7 displays the measured interferometer wavelength shifts $(\Delta \lambda)$ and error on temperature $(\mathrm{T})$. As the temperature increases, the interferometer wavelength shifts to longer wavelength. A third-order polynomial was used to fit the wavelength shifts across the entire calibration range. The average sensitivity of the device is $\sim 17 \mathrm{pm} /{ }^{\circ} \mathrm{C}$, which is very close to the theoretical result. Higher sensitivity can be obtained by optimizing the profile of the SMF-TT or using special fiber taper with higher thermo-optics coefficient (Kou et al., 2010b).

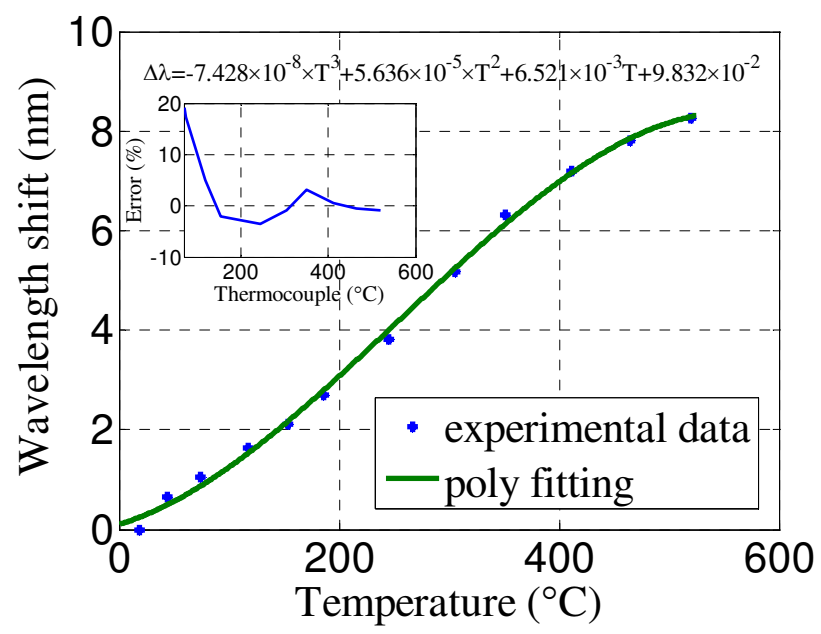

Fig. 7. Dependence of the measured wavelength shift on temperature. The asterisk represents the measured results while the solid line is the fitting result. The inset shows the dependence of error on temperature (Kou et al., 2010b). Reprinted with permission. Copyright 2010 Optical Society of America 


\subsection{FIB machined FPMI for refractive index sensing}

The FPMI also can be employed as a refractive index sensor. Figure 8 shows the SEM picture of another FPMI with a micro-notch cavity from the side view. The cavity is $3.50 \mu \mathrm{m}$ long and $2.94 \mu \mathrm{m}$ high, located at the position with the local radius $r=2.4 \mu \mathrm{m}$.

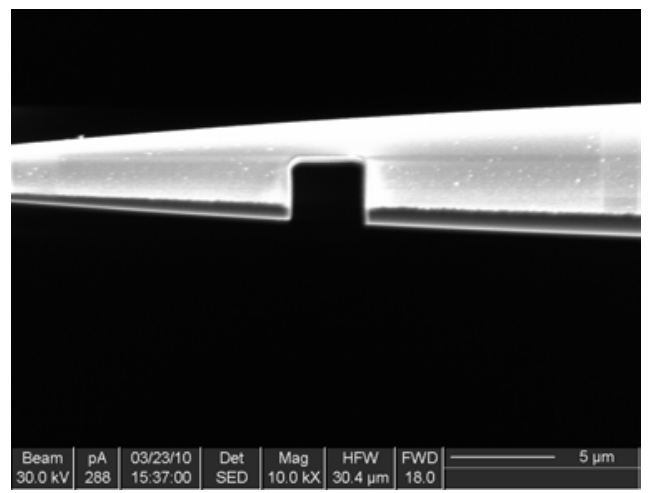

Fig. 8. SEM image of the micro-notch cavity from the side view (Kou et al., 2010a). Reprinted with permission. Copyright 2010 Optical Society of America

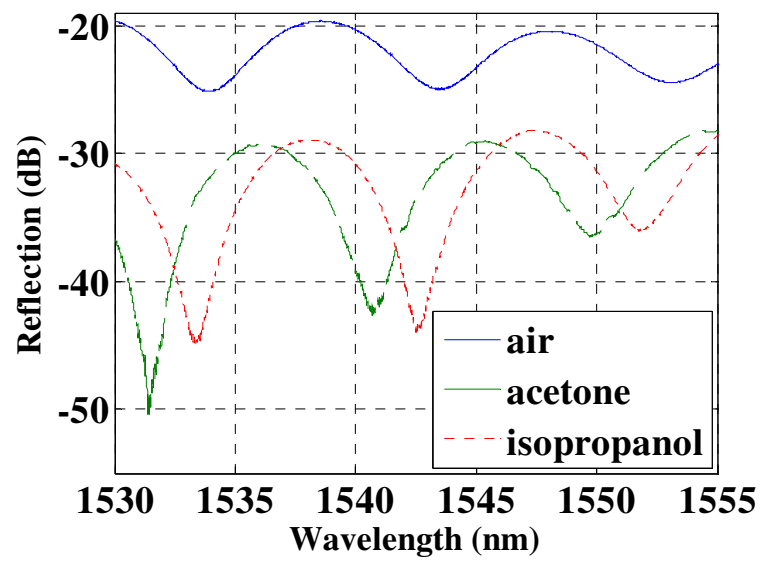

Fig. 9. Interference spectra of the MPRI device in air (solid line), acetone (dashed line) and isopropanol (dotted line), at room temperature $\left(25^{\circ} \mathrm{C}\right)$ (Kou et al., 2010a). Reprinted with permission. Copyright 2010 Optical Society of America

The reflective Interference spectrum is measured with the same setup as shown in Fig. 2. Figure 9 shows the interference spectra of the MPRI in air, acetone and isopropanol at room temperature $\left(25^{\circ} \mathrm{C}\right)$. The interference spectrum indicates a fringe visibility maximum of $\sim 20$ $\mathrm{dB}$, which is much higher than those of typical MCFPIs in liquids.

The performance of resonant or interferometer refractive index sensors can be evaluated by using the sensitivity $S_{R}$, which is defined as the magnitude in shift of the resonant wavelength divided by the change in refractive index of the analyte. The sensitivity was 
measured by inserting the sensor in mixtures of isopropanol and acetone. These solutions were chosen with the objective of simulating aqueous solutions, having a refractive index in the region around 1.33 at a wavelength of $1550 \mathrm{~nm}$. The ratio was increased by adding small calibrated quantities of isopropyl to the solution at a position far from the sensor. The refractive indexes of pure isopropyl and acetone at $1550 \mathrm{~nm}$ are 1.3739 and 1.3577 respectively (Wei et al., 2008).

Figure 10 displays the shifted spectral wavelength as a function of the liquid mixture refractive index. The asterisks represent the measurement results while the solid line is the best-fitting. As the refractive index increases, the spectrum shows a red-shift. The sensitivity of the device is $110 \mathrm{~nm} / \mathrm{RIU}$ (refractive index unit) according to Fig. 11. Higher sensitivity can be obtained by optimizing the profile of the microfiber taper probe. Due to its small size, fiber-probe structure, all fiber connection, linear response, low-cost, easy fabrication and high sensitivity, MPRI devices are promising in various chemical and biological applications. It even may offer fantastic potentials sensing inside sub-wavelength liquid droplets, bubbles or biocells because of its unique probe structure and possible smaller size (Kou et al., 2010a).

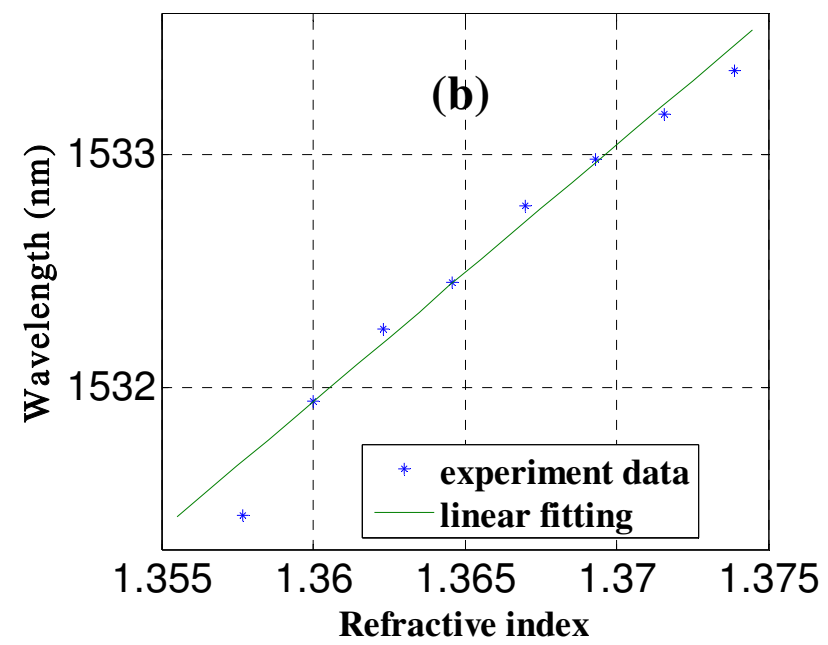

Fig. 10. The shifted spectral wavelength as a function of the liquid mixture refractive index. The asterisks represent the measured results while the solid line is the fitting results (Kou et al., 2010a). Reprinted with permission. Copyright 2010 Optical Society of America

\section{FIB machined TFT micro-grating}

Since their discovery in 1978 (Hill et al., 1978), optical fiber gratings have found a variety of applications in telecom and sensing because of their relatively low cost, inherent selfreferencing and multiplexing/demultiplexing capabilities. Over the last two decades, fiber gratings including fiber Bragg gratings (FBGs) and long-period gratings (LPGs) have been manufactured mainly by modifying the core refractive index using interferometric or pointby-point techniques; most of interferometric techniques use a phase mask and an ultraviolet (UV) laser (Hill et al., 1993) (typically excimer or frequency doubled $\mathrm{Ar}^{+}$ion) or femtosecond 
lasers (near IR or UV). Gratings based on surface etched corrugations have also been demonstrated in etched fibers using photolithographic techniques (Lin \& Wang, 2001). However all these gratings fabricated in thick fibers have weak refractive index modulations $\left(\Delta \mathrm{n}_{\text {mod }} \sim 10^{-4}-10^{-3}\right)$ and the related grating lengths are of the order of several millimeters. To reduce the grating length, strong refractive index modulations $\left(\Delta \mathrm{n}_{\bmod }>10^{-2}\right)$ are necessary. Strong $\Delta \mathrm{n}_{\text {mod }}$ can be obtained by alternating layers of different materials, one of which can be air. Although this process in normal optical fibers imposes the removal of large amounts of material (the propagating mode is confined at a depth $>50 \mu \mathrm{m}$ from the fiber surface), in fiber tapers and tips it requires the removal of small amounts of matter because the propagating mode is confined to the silica/air interface. A few techniques have been proposed for the fabrication of gratings in microfibers, including photorefractive inscription using $\mathrm{CO}_{2}$ lasers (Xuan et al., 2009), femtosecond lasers (Martinez et al., 2005; Xuan et al., 2010) and wrapping a microfiber on a microstructured rod (Xu et al., 2009, 2010). None of them produced strong and short Bragg gratings. In some cases extra polymer coatings are needed, while in others the use of $\mathrm{CO}_{2}$ lasers implies that the grating length is still long (it only can be used to write long period gratings (LPG) or high-order FBG). As a consequence, devices based on gratings tend to have a sizeable length: typically FBGs have lengths in the order of few millimetres. FIB technique provides a powerful way to mill the microfiber with directly and flexibly without a mask and realize compact micro-gratings (tens of micrometers) with colourful structure in the surface. Such small size and unique structure grating offer great potentials for a lot of sensing applications such as high temperature and refractive index sensing with the advantages of fast-response, ability to work in harsh environments and occupying little space.

\subsection{FIB machined micro-grating for temperature sensing}

Figure 11 shows an SEM micrograph of an ultra-short second-order TFT micro-grating (TFT$\mathrm{MG}$ ). The grating has 11 shallow corrugations with period $\Lambda=1.1 \mu \mathrm{m}$, providing a total length of $\sim 12 \mu \mathrm{m}$, two orders of magnitude shorter than FBGs fabricated in conventional optical fiber. Each notch is $\sim 1.6 \mu \mathrm{m}$ deep and $\sim 0.6 \mu \mathrm{m}$ long. The average radius at the position where the notches are located is $\sim 2.7 \mu \mathrm{m}$. The Bragg wavelength of the grating can be calculated from $\lambda_{\mathrm{g}}=2 \mathrm{n}_{\text {eff }} \Lambda / \mathrm{m}$, where $\mathrm{n}_{\mathrm{eff}}$ is the mode effective refractive index in the equivalent unperturbed geometry, $\Lambda$ is the period and $\mathrm{m}$ is the Bragg order. Unlike conventional circularly-symmetric FBGs, this nanostructured TFT-MG has asymmetric periodic corrugations. The modal field and $\mathrm{n}_{\mathrm{eff}}$ in the nano-structured TFT can be derived analytically from the bare TFT using the method developed by W. Streifer, which considers an equivalent structure, obtained by shifting the boundary between air and silica to compensate for the different geometry. Figure 12 shows the cross-sections of an un-etched fiber, an etched fiber and the equivalent unperturbed geometry with this method, which shifts the boundary between air and silica to compensate for the different geometry (Streifer et al., 1975, 1978), respectively. The effective groove height $h_{\text {eff }}$ of the equivalent unperturbed geometry satisfies (Kou et al., 2011a):

$$
\left\{\begin{array}{c}
(1-\tau)\left(\theta_{g}-\sin \theta_{g} \cos \theta_{g}\right)=\theta_{e f f}-\sin \theta_{e f f} \cos \theta_{e f f} \\
\theta_{g}=\arccos \left\{\left(r-h_{g}\right) / r\right\} \\
\theta_{e f f}=\arccos \left\{\left(r-h_{e f f}\right) / r\right\}
\end{array}\right.
$$


where $\tau$ is the grating duty cycle, $h_{g}$ is the groove height and $r$ is the fiber radius, respectively. In our device, $r=2.7 \mu \mathrm{m}, \tau=0.33$ and $h_{g}=1.6 \mu \mathrm{m}$, we find $h_{\text {eff }}=1.2 \mu \mathrm{m}$ by solving Eq. 5 and $n_{\text {eff }}=1.428$ by utilizing a finite element method. Thus, the Bragg wavelength is $1571 \mathrm{~nm}$. It agrees well with the following experimental results.

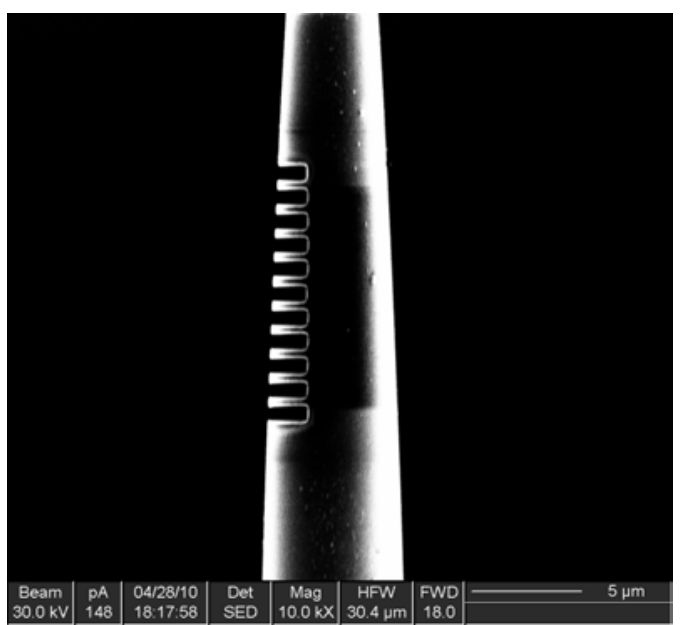

Fig. 11. SEM picture of the nanostructured TFT-MG. The grating has 11 notches and a total length of $\sim 12 \mu \mathrm{m}$. The notch length and depth are $\sim 0.6 \mu \mathrm{m}$ and $\sim 1.6 \mu \mathrm{m}$, respectively. The grating period is $\Lambda \sim 1.1 \mu \mathrm{m}$ (Feng et al., 2011).

(a)

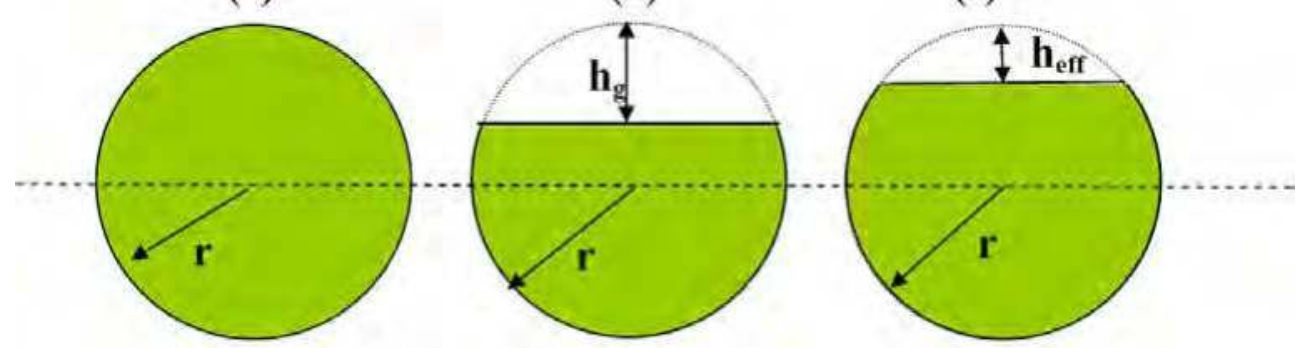

Original microfiber
Machined microfiber (b)

Equivalent unperturbed geometry

Fig. 12. The cross-sections of un-etched fiber (a), etched fiber (b) and equivalent unperturbed geometry (c), respectively. $h_{g}$ is the groove height and $h_{\text {eff }}$ is effective height (Kou et al., 2011a).

The reflective spectral response of the TFT-MG in Fig. 10 is measured with the setup shown in Fig. 2. We characterize the thermal response of the TFT-MG by heating it up in a microfurnace from room temperature $\left(20^{\circ} \mathrm{C}\right)$ to $230{ }^{\circ} \mathrm{C}$. The spectrum and temperature are recorded when both of them are stable for several minutes. 
The interference spectra of the TFT-MG at different temperatures $\left(23^{\circ} \mathrm{C}, 47^{\circ} \mathrm{C}, 104^{\circ} \mathrm{C}, 153^{\circ} \mathrm{C}\right.$ and $228^{\circ} \mathrm{C}$ ) are shown in Fig. 13. The Bragg wavelength is $\sim 1570 \mathrm{~nm}$, in agreement with our theoretical calculation. The spectra indicate a reflection peak-to-trough ratio around 1570 $\mathrm{nm}$ of $\sim 10 \mathrm{~dB}$ at the Bragg wavelength which is achieved with as few as 11 periods and is similar with or even better than some other long length fiber gratings, enough for sensing applications.

The temperature sensitivity $\mathrm{S}_{\mathrm{T}}$ is defined as (Kou et al., 2011a):

$$
S_{T}=\frac{d \lambda_{g}}{d T}=\frac{2}{m}\left(\sigma_{T} \Lambda \frac{\partial n_{\text {eff }}}{\partial n_{\text {silica }}}+\Lambda \alpha_{T} n_{\text {eff }}+r \Lambda \alpha_{T} \frac{\partial n_{\text {eff }}}{\partial r}\right)
$$

where $\sigma_{\mathrm{T}}\left(1.4 \times 10-5 /{ }^{\circ} \mathrm{C}\right)$ is the thermo-optic coefficient and $\mathrm{a}_{\mathrm{T}}\left(5.5 \times 10-7 /{ }^{\circ} \mathrm{C}\right)$ is the thermal expansion coefficient. According to our calculations, the first part is about $15 \sim 20 \mathrm{pm} /{ }^{\circ} \mathrm{C}$ and dominates in temperature sensing. Thermal expansion effect (the second and third parts of Eq. 6) contributes little to the total sensitivity $(<6 \%)$, mainly due to the low thermal expansion coefficient of silica. Moreover, in the first part of Eq. 2, $\partial n_{\text {eff }} / \partial n_{\text {silica }}$ is nearly 1 and does not change much with the microfiber diameter, which means that the most efficient method to increase thermal sensitivity is to use fiber with higher thermo-optics coefficient.

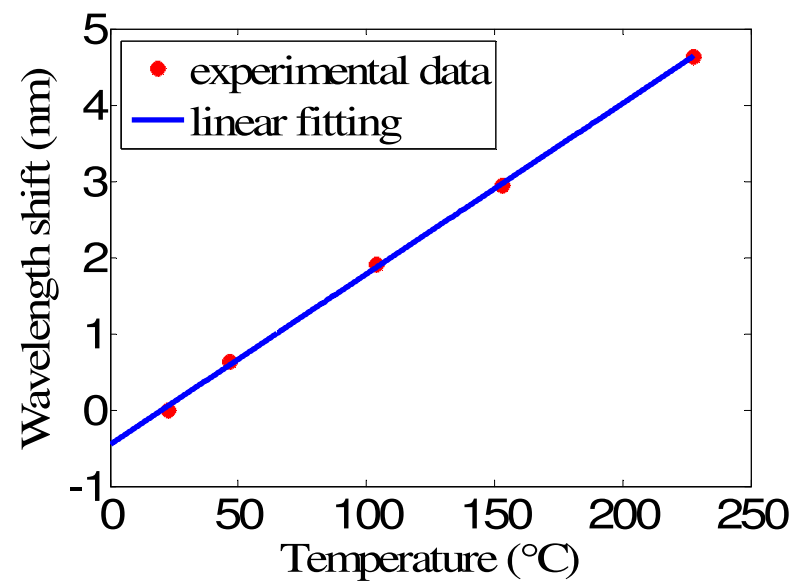

Fig. 13. Dependence of the measured wavelength shift on temperature. The asterisk represents the measured results while the solid line is the linear fitting result (Feng et al., 2011).

Figure 13 displays the measured resonant wavelength shifts $(\Delta \lambda)$ on temperature $(\mathrm{T})$. As the temperature increases, the interference wavelength shifts to longer wavelength. The average sensitivity of the device is $\sim 22 \mathrm{pm} /{ }^{\circ} \mathrm{C}$, which is very close to the theoretical result, higher than or similar with previous fiber grating sensors. Higher sensitivity can be obtained by use special fiber taper with higher thermo-optics coefficient.

First-order micro-grating $(\mathrm{m}=1)$ with smaller period also can be fabricated in TFT by FIB. Figure 14 shows the SEM photography of a first-order TFT-MG from the side view. The grating has shallow corrugations of period $\Lambda=600 \mathrm{~nm}$ with 61 periods. The total length is 
about $36.6 \mu \mathrm{m}$, which is extremely short. Every groove is $200 \mathrm{~nm}$ in depth, located at the position with the local radius around $r=3.25 \mu \mathrm{m}$. The resonant spectra of the TFT-MG at different temperatures are shown in Fig. 15. The Bragg wavelength is $\sim 1550 \mathrm{~nm}$, with excited higher order mode as deduced from our theoretical calculation. The spectra indicate an extinction ratio of $\sim 11 \mathrm{~dB}$ at the Bragg wavelength which is achieved with a $36.6 \mathrm{um}$ long Bragg grating. The average temperature sensitivity of the device from room temperature to around $500{ }^{\circ} \mathrm{C}$ is $\sim 20 \mathrm{pm} /{ }^{\circ} \mathrm{C}$ as shown in Fig. 15 (b), which is similar with the second-order TFT-MG. It is reasonable because the main thermal contribution is from the thermo-optic effect (Kou et al., 2011a).
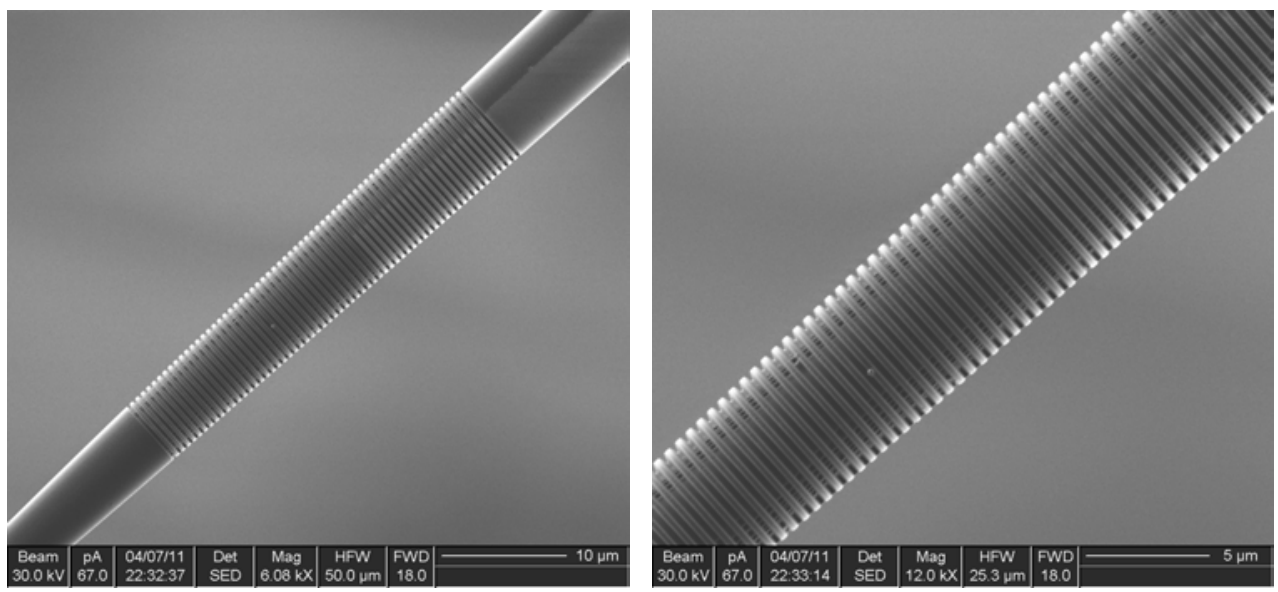

Fig. 14. Left: FIB picture of the TFPG with 61 periods $(\sim 36.6 \mu \mathrm{m}$ in length and $\Lambda=600 \mathrm{~nm})$. Right: magnified picture of the grating (Kou et al., 2011a). Reprinted with permission. Copyright 2010 Optical Society of America
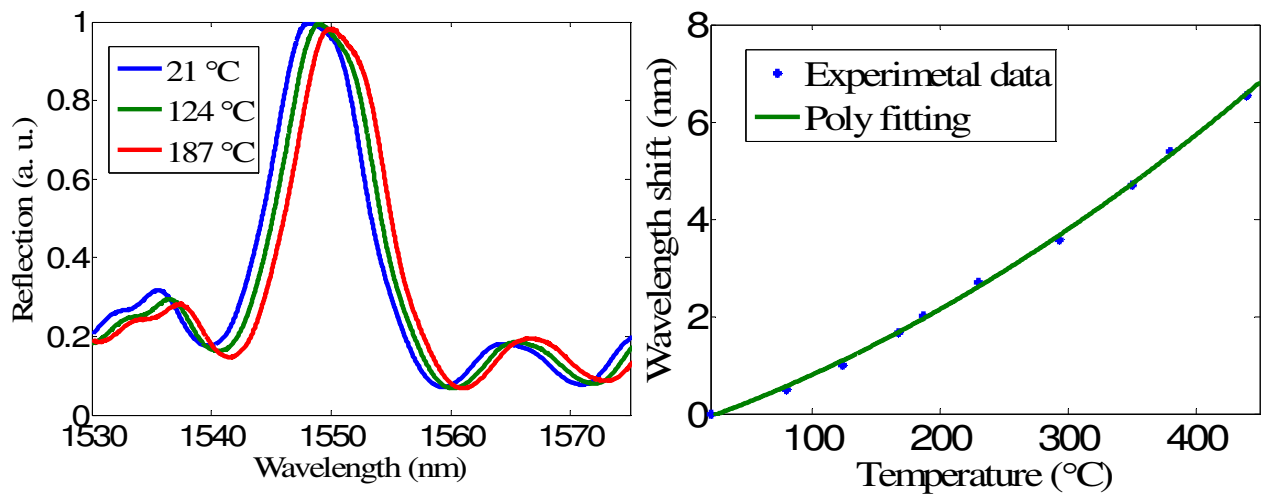

Fig. 15. (a) Reflection spectra of the first-order TFT-MG in air at different temperatures. (b) Dependence of the measured wavelength shift on temperature. The asterisk represents the measured results while the solid line is the linear fitting result (Kou et al., 2011a).

Reprinted with permission. Copyright 2010 Optical Society of America 


\subsection{FIB machined metal-dielectric-hybrid micro-grating for refractive index sensing}

Conventional FBGs have been extensively developed to measure the temperature, pressure or stress. But it is scarcely used to measure the environmental refractive index variation because there is almost no evanescent field penetrating outside of a standard $125 \mu \mathrm{m}$ diameter FBG. TFT-MG may overcome the drawback with the available evanescence field interacting with the outer environments. The sensitivity of a pure-silica TFT-MG with the diameter of several micrometers is about tens of $\mathrm{nm} / \mathrm{RIU}$. By inducing metal-cladding, more cladding modes are possible to be excited and higher sensitivity can be obtained, which is so called grating-assisted surface plasmon-polariton (SPP)-like grating sensor (Nemova \& Kashyap, 2006).

Figure 16 shows the SEM picture of a metal-dielectric-hybrid TFT-MG (MD-TFT-MG) by FIB milling. The fabrication process is similar with those mentioned ones above. But the fiber tip is coated with a gold layer with thickness of $30 \mathrm{~nm}$ on one side by magnetron sputtering and it is kept all the way throughout the experiment. We choose gold due to its relatively low absorption in the infrared and inertness to oxidation when exposed in air. Then a grating is fabricated by FIB milling at the fiber tip with local radius of $\sim 3 \mu \mathrm{m}$. The grating has shallow corrugations of period $\Lambda=578 \mathrm{~nm}$ with 17 periods. The total length is about $10 \mu \mathrm{m}$, which is extremely short with local radius of $\sim 3 \mu \mathrm{m}$.

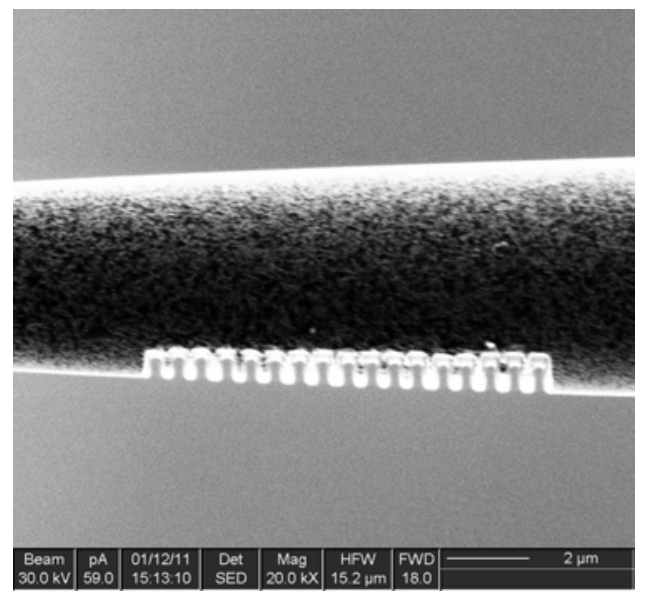

Fig. 16. SEM picture of the metal-dielectric-hybrid fiber tip grating $(\sim 10 \mu \mathrm{m}$ in length and $\Lambda=578 \mathrm{~nm}$ ). Right: magnified picture of the grating (Kou et al., 2011b).

Optical characterization of the MD-TFT-MG in Fig. 16 is performed using the same setup as shown in Fig. 2. Figure 17 shows the reflection spectra of the MD-TFT-MG in air, acetone, and isopropanol, respectively. The extinction ratio is about $\sim 10 \mathrm{~dB}$. There are several valleys and peaks with different characteristics in the spectral range of $\sim 100 \mathrm{~nm}$. They shift when the outer environment changes from acetone to isopropanol. However, these valleys and peaks show larger shifts at longer wavelengths, while those at shorter wavelength region shift much less and almost stop at specific wavelengths. This unique response to outer liquid refractive index comes from the fact that the reflected light can be coupled to different modes. In the micrometer-diameter metal-dielectric-hybrid TFT, several modes are probably excited with similar propagation constant because of the metal cladding. Some modes are well confined in the tip and have negligible field overlap with the liquid while some modes 
are not. The different valleys and peaks correspond to the coupling between these different forward and backward propagating modes, with different response properties for the outer environment.

The reflection resonant condition for the grating is:

$$
\frac{2 \pi}{\lambda_{g}}\left[n_{f}+n_{b}\right]=\frac{2 \pi}{\Lambda}
$$

where $n_{f}$ and $n_{b}$ are the effective indices of the forward and backward modes, respectively. For simplicity, we assume a theoretical model to explain our experimental results which is simple and not perfectly matched with the experiment but can give the fundamental mechanism of the device. Within the model, the microfiber is $6 \mu \mathrm{m}$ in diameter with uniform metal cladding (20 $\mathrm{nm}$ in thickness). However, the real device is much more complicated, with nonuniform metal cladding and diameter. And if an asymmetrical mode field lies mainly near the grating, leading to a larger modal overlap with the grating, it may result in a higher sensitivity. Figure 3 shows the calculation on the effective index of one cladding mode and one core mode as a function of outer liquid refractive index $\mathrm{n}_{\mathrm{l}}$. Due to the existence of the metal layer, the cladding mode has a larger effective index (corresponding to long resonant wavelength) than that of the core mode (corresponding to short resonant wavelength) and has a larger overlap with the taper surface and the outside environment, leading to a higher sensitivity to the surrounding medium which is in coincidence with the spectra of Fig. 2.

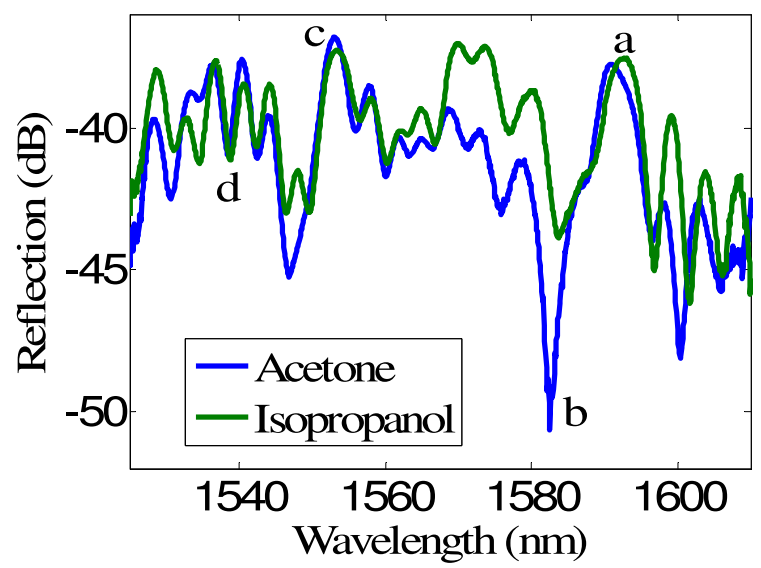

Fig. 17. Measured reflection spectra of the FTG when immersed in acetone and isopropanol (Kou et al., 2011b).

The performance of resonant refractive index sensors can be evaluated by using sensitivity S, which is defined as the magnitude in shift of the resonant wavelength divided by the change in refractive index of the analyte. In our experiment, the sensitivity is measured by inserting the sensor in a beaker containing mixtures of isopropanol and acetone, where the isopropanol component has the following ratios: $0,1 / 7,2 / 7,3 / 7,4 / 75 / 7,6 / 7$, and 1 (Kou et al., 2011b). Figure 18 displays measured resonant wavelength shifts of several peaks and valleys and fitting of this FTG on the liquid refractive index (a, b, c, d as marked in Fig. 2, a and c are 
peaks, $b$ and $d$ are valleys). As the refractive index increases, the resonant wavelength shifts to longer wavelength. The sensitivities of different modes change severely. It can be as high as $125 \mathrm{~nm} / \mathrm{RIU}$ (peak a) or as low as $7 \mathrm{~nm} / \mathrm{RIU}$ (valley d). For peak a (or valley b), both the resonant wavelength and sensitivity are larger than those of peak c (or valley $\mathrm{d}$ ). According to our theoretical calculation, we believe peak a (or valley $b$ ) corresponds to cladding mode while peak c (or valley d) is core mode. The smallest sensitivity can be further decreased to nearly zero by optimizing the tip grating profile and metal coating. Because of many different properties on the outer liquid refractive index, the metal-dielectric-hybrid FTG can be applied as a multi-parameter sensor and the index-insensitive channel can be used to simultaneously measure temperature, pressure, and so on (Kou et al., 2011b).

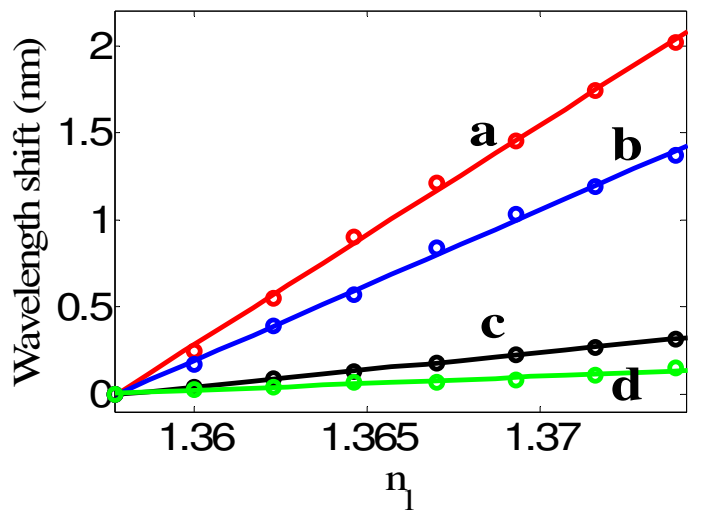

Fig. 18. Dependence of wavelength shift on outer liquid refractive index $n_{1}$. The asterisks represent the experimental results with the solid line of linear fitting (Kou et al., 2010b).

\section{Conclusion}

In this chapter, FIB machined TFT based micro-devices including interferometers and gratings are demonstrated. Being a very flexible, mask-less, direct write process, FIB milling is perfect for carving nanoscale geometries precisely in microfibers. Various miniature fiber devices can be realized and they show great potential in sensing with the unique geometry and size. The sensitivity such as of temperature or refractive index can't increase too much because it mainly depends on the fiber materials and size. But the ultra-small size is attractive for some special application, in particular for detecting small-size objects. Some novel geometry is possible to be realized in microfiber such as an inline-microring, a slot-microfiber etc.

\section{Acknowledgment}

This work is supported by National 973 program under contract No. 2010CB327803, 2012CB921803 and 2011CBA00200, NSFC program No. 11074117 and 60977039. The authors also acknowledge the support from the Priority Academic Program Development of Jiangsu (PAPD), and the Fundamental Research Funds for the Central Universities.

\section{References}

Agrawal, G. P. (2002). Fiber-optic communication systems. New York, Wiley-Interscience. 
Bhatia, V., Murphy, K. A., Claus, R. O., Jones, M. E., Grace, J. L., Tran, T. A. \& Greene, J. A. (1996). Optical fibre based absolute extrinsic Fabry-Perot interferometric sensing system. Measurement Science E Technology, Vol. 7, No. 1, (1996), pp. 58-61

Brambilla, G. (2010). Optical fibre nanowires and microwires: a review. Journal of Optics, Vol. 12, No. 4, (2010), pp. 043001

Brambilla, G., Finazzi, V. \& Richardson, D. (2004). Ultra-low-loss optical fiber nanotapers. Optics Express, Vol. 12, No. 10, (2004), pp. 2258-2263

Brambilla, G., Koizumi, E., Feng, X. \& Richardson, D. J. (2005). Compound-glass optical nanowires. Electronics Letters, Vol. 41, No. 7, (2005), pp. 400-402

Brambilla, G. \& Xu, F. (2007). Adiabatic submicrometric tapers for optical tweezers. Electronics Letters, Vol. 43, No. 4, (2007), pp. 204-206

Choi, H. Y., Park, K. S., Park, S. J., Paek, U.-C., Lee, B. H. \& Choi, E. S. (2008). Miniature fiber-optic high temperature sensor based on a hybrid structured Fabry-Perot interferometer. Optics Letters, Vol. 33, No. 21, (2008), pp. 2455-2457

Daniel, J. H., Moore, D. F. \& Walker, J. F. (1998). Focused ion beams for microfabrication. Engineering Science and Education Journal, Vol. 7, No. 2, (1998), pp. 53-56

Deng, Z. F., Yenilmez, E., Reilein, A., Leu, J., Dai, H. J. \& Moler, K. A. (2006). Nanotube manipulation with focused ion beam. Applied Physics Letters, Vol. 88, No. 2, (2006),

Feng, J., Ding, M., Kou, J.-1., Xu, F. \& Lu, Y.-q. (2011). An optical fiber tip micro-grating thermometer. IEEE Photonics Journal, Vol. 3, No. 5, (2011), pp. 810-814,

Hill, K. O., Fujii, Y., Johnson, D. C. \& Kawasaki, B. S. (1978). Photosensitivity in Optical Fiber Waveguides - Application to Reflection Filter Fabrication. Applied Physics Letters, Vol. 32, No. 10, (1978), pp. 647-649

Hill, K. O., Malo, B., Bilodeau, F., Johnson, D. C. \& Albert, J. (1993). Bragg Gratings Fabricated in Monomode Photosensitive Optical-Fiber by UV Exposure through a Phase Mask. Applied Physics Letters, Vol. 63, No. 3, (1993), pp. 424-424

Hofmann, S., Cantoro, M., Kaempgen, M., Kang, D. J., Golovko, V. B., Li, H. W., Yang, Z., Geng, J., Huck, W. T. S., Johnson, B. F. G., Roth, S. \& Robertson, J. (2005). Catalyst patterning methods for surface-bound chemical vapor deposition of carbon nanotubes. Applied Physics A: Materials Science \& Processing, Vol. 81, No. 8, (2005), pp. 1559-1567

Hopman, W. C. L., Ay, F. \& Ridder, R. M. d. (2008). Focused ion beam milling strategy for submicrometer holes in silicon. Workshop FIB for Photonics, Eindhoven, the Netherlands. http:/ / en.wikipedia.org/wiki/Focused_ion_beam

Jeon, J., Floresca, H. C. \& Kim, M. J. (2010). Fabrication of complex three-dimensional nanostructures using focused ion beam and nanomanipulation Journal of Vacuum Science E Technology B, Vol. 28, No. 3, (2010), pp. 549-553

Kou, J.-1., Feng, J., Wang, Q.-j., Xu, F. \& Lu, Y.-q. (2010a). Microfiber-probe-based ultrasmall interferometric sensor. Optics Letters, Vol. 35, No. 13, (2010a), pp. 2308-2310

Kou, J.-l., Feng, J., Ye, L., Xu, F. \& Lu, Y.-q. (2010b). Miniaturized fiber taper reflective interferometer for high temperature measurement. Optics Express, Vol. 18, No. 13, (2010b), pp. 14245-14250

Kou, J.-1., Qiu, S.-j., Xu, F. \& Lu, Y.-q. (2011a). Demonstration of a compact temperature sensor based on first-order Bragg grating in a tapered fiber probe. Optics Express, Vol. 19, No. 19, (2011a), pp. 18452-18457

Kou, J.-1., Qiu, S.-j., Yuan, Y., Zhao, G., Xu, F. \& Lu, Y.-q. (2011b). Miniaturized Metaldielectric-hybrid Fiber Tip Grating for Refractive Index Sensing. IEEE Photonics Technology Letters, Vol. 23, No. 22, (2011), pp. 1712-1714,(2011b), 
Liang, T., Stivers, A., Livengood, R., Yan, P. Y., Zhang, G. J. \& Lo, F. C. (2000). Progress in extreme ultraviolet mask repair using a focused ion beam. Journal of Vacuum Science E Technology B, Vol. 18, No. 6, (2000), pp. 3216-3220

Lin, C. Y. \& Wang, L. A. (2001). A wavelength- and loss-tunable band-rejection filter based on corrugated long-period fiber grating. IEEE Photonics Technology Letters, Vol. 13, No. 4, (2001), pp. 332-334

Liu, K., Soskov, A., Scipioni, L., Bassom, N., Sijbrandij, S. \& Smith, G. (2006). Electrical breakthrough effect for end pointing in 90 and $45 \mathrm{~nm}$ node circuit edit. Applied Physics Letters, Vol. 88, No. 12, (2006), pp. 124104

Love, J. D., Henry, W. M., Stewart, W. J., Black, R. J., Lacroix, S. \& Gonthier, F. (1991). Tapered single-mode fibres and devices. I. Adiabaticity criteria. Optoelectronics, IEE Proceedings Journal, Vol. 138, No. 5, (1991), pp. 343-354

Martinez, A., Khrushchev, I. Y. \& Bennion, I. (2005). Thermal properties of fibre Bragg gratings inscribed point-by-point by infrared femtosecond laser. Electronics Letters, Vol. 41, No. 4, (2005), pp. 176-178

Nemova, G. \& Kashyap, R. (2006). Fiber-Bragg-grating-assisted surface plasmon-polariton sensor. Optics Leters, Vol. 31, No. 14, (2006), pp. 2118-2120

Rao, Y.-J., Deng, M., Duan, D.-W., Yang, X.-C., Zhu, T. \& Cheng, G.-H. (2007). Micro FabryPerot interferometers in silica fibers machined by femtosecond laser. Optics Express, Vol. 15, No. 21, (2007), pp. 14123-14128

Sirkis, J. S., Brennan, D. D., Putman, M. A., Berkoff, T. A., Kersey, A. D. \& Friebele, E. J. (1993). In-line fiber ealon for strain measurement. Optics Letters, Vol. 18, No. 22, (1993), pp. 1973-1975

Streifer, W. \& Hardy, A. (1978). Analysis of two-dimensional waveguides with misaligned or curved gratings. IEEE Journal of Quantum Electronics, Vol. 14, No. 12, (1978), pp. 935-943

Streifer, W., Scifres, D. \& Burnham, R. (1975). Coupling coefficients for distributed feedback single- and double-heterostructure diode lasers. IEEE Journal of Quantum Electronics, Vol. 11, No. 11, (1975), pp. 867-873

Terris, B., Thomson, T. \& Hu, G. (2007). Patterned media for future magnetic data storage. Microsystem Technologies, Vol. 13, No. 2, (2007), pp. 189-196

Tong, L. M., Gattass, R. R., Ashcom, J. B., He, S. L., Lou, J. Y., Shen, M. Y., Maxwell, I. \& Mazur, E. (2003). Subwavelength-diameter silica wires for low-loss optical wave guiding. Nature, Vol. 426, No. 6968, (2003), pp. 816-819

Villatoro, J., Finazzi, V., Coviello, G. \& Pruneri, V. (2009). Photonic-crystal-fiber-enabled microFabry?Perot interferometer. Optics Letters, Vol. 34, No. 16, (2009), pp. 2441-2443

Wei, T., Han, Y., Li, Y., Tsai, H.-L. \& Xiao, H. (2008). Temperature-insensitive miniaturized fiber inline Fabry-Perot interferometer for highly sensitive refractive index measurement. Optics Express, Vol. 16, No. 8, (2008), pp. 5764-5769

Xu, F., Brambilla, G., Feng, J. \& Lu, Y.-Q. (2010). A Microfiber Bragg Grating Based on a Microstructured Rod: A Proposal. IEEE Photonics Technology Letters, Vol. 22, No. 4, (2010), pp. 218-220

Xu, F., Brambilla, G. \& Lu, Y. (2009). A microfluidic refractometric sensor based on gratings in optical fibre microwires. Optics Express, Vol. 17, No. 23, (2009), pp. 20866-20871

Xuan, H., Jin, W. \& Liu, S. (2010). Long-period gratings in wavelength-scale microfibers. Optics Letters, Vol. 35, No. 1, (2010), pp. 85-87

Xuan, H., Jin, W. \& Zhang, M. (2009). $\mathrm{CO}_{2}$ laser induced long period gratings in optical microfibers. Optics Express, Vol. 17, No. 24, (2009), pp. 21882-21890 


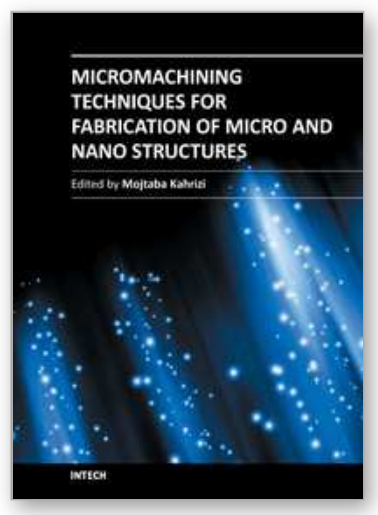

\section{Micromachining Techniques for Fabrication of Micro and Nano Structures}

Edited by Dr. Mojtaba Kahrizi

ISBN 978-953-307-906-6

Hard cover, 300 pages

Publisher InTech

Published online 03, February, 2012

Published in print edition February, 2012

Micromachining is used to fabricate three-dimensional microstructures and it is the foundation of a technology called Micro-Electro-Mechanical-Systems (MEMS). Bulk micromachining and surface micromachining are two major categories (among others) in this field. This book presents advances in micromachining technology. For this, we have gathered review articles related to various techniques and methods of micro/nano fabrications, like focused ion beams, laser ablation, and several other specialized techniques, from esteemed researchers and scientists around the world. Each chapter gives a complete description of a specific micromachining method, design, associate analytical works, experimental set-up, and the final fabricated devices, followed by many references related to this field of research available in other literature. Due to the multidisciplinary nature of this technology, the collection of articles presented here can be used by scientists and researchers in the disciplines of engineering, materials sciences, physics, and chemistry.

\section{How to reference}

In order to correctly reference this scholarly work, feel free to copy and paste the following:

Fei Xu, Jun-long Kou, Yan-qing Lu and Wei Hu (2012). Miniature Engineered Tapered Fiber Tip Devices by Focused Ion Beam Micromachining, Micromachining Techniques for Fabrication of Micro and Nano Structures, Dr. Mojtaba Kahrizi (Ed.), ISBN: 978-953-307-906-6, InTech, Available from:

http://www.intechopen.com/books/micromachining-techniques-for-fabrication-of-micro-and-nanostructures/miniature-engineered-tapered-fiber-tip-devices-by-focused-ion-beam-micromachining

\section{INTECH}

open science | open minds

\section{InTech Europe}

University Campus STeP Ri

Slavka Krautzeka 83/A

51000 Rijeka, Croatia

Phone: +385 (51) 770447

Fax: +385 (51) 686166

www.intechopen.com

\section{InTech China}

Unit 405, Office Block, Hotel Equatorial Shanghai

No.65, Yan An Road (West), Shanghai, 200040, China

中国上海市延安西路65号上海国际贵都大饭店办公楼 405 单元

Phone: +86-21-62489820

Fax: $+86-21-62489821$ 
(C) 2012 The Author(s). Licensee IntechOpen. This is an open access article distributed under the terms of the Creative Commons Attribution 3.0 License, which permits unrestricted use, distribution, and reproduction in any medium, provided the original work is properly cited. 